stage ( 1 and 2 ) while 21 cases had advanced disease ( 3 and 4 ). $48.78 \%$ cases were primarily treated with surgery, $26.83 \%$ with radiotherapy, $7.3 \%$ with chemotherapy and $17.07 \%$ with combined chemoradiation. $78 \%$ of surgically treated cases had mean survival of 5 years. Mean survival of 1 year was recorded in advanced disease cases. Limitation of the study was poor follow up after treatment.

Conclusion: Incidence of vulvar cancer is significantly high in multiparous and postmenopausal women. Surgical treatment is the best option in early stage of disease (stage I and II) and gives high survival rates while advanced disease treated with chemoradiation has poor survival.

\section{Miscellaneous: Oral Abstract}

\author{
Association of TNF- $\alpha-$ rs 281865419 polymorphism with \\ reproductive tract infections in Indian population \\ Vineeta Sharma ${ }^{1,2}$, Pallavi Singhal ${ }^{1}$, \\ Anoop Kumar', V. G. Ramachandran², Shukla Das², \\ Mausumi Bharadwaj ${ }^{1}$ \\ ${ }^{1}$ Division of Molecular Genetics and Biochemistry, Institute \\ of Cytology and Preventive Oncology, Noida, Uttar Pradesh, \\ ${ }^{2}$ Department of Microbiology, University College of Medical \\ Science, Delhi University, New Delhi, India
}

Aim: To investigate the presence of reproductive tract infections (RTIs) in symptomatic and asymptomatic women in North India and association of SNPs in TNF $\alpha$ gene (rs-281865419 C/T) with susceptibility to these RTIs. Methods: We collected 100 symptomatic (cases) and 100 asymptomatic women (controls) samples and screened them for RTIs. Then genotyping of TNF- $\alpha$ gene was performed by PCR-RFLP.

Results: Among cases the frequencies of RTIs infection is higher than control. The prevalence of HPV, C. trachomatis, T. vaginalis, Bacterial vaginosis and $N$. gonorrhoeae are $28 \%$ and $6 \% ; 11 \%, 32 \%$ respectively while in controls it was $5 \%, 2 \%, 1 \%$ and $8 \%$ and $1 \%$. In the present study we found that the frequency of wild homozygous genotype (TT) was lower in cases $30 \%(6 / 20)$ as compared to controls $60 \%(12 / 20)$. The frequency of the heterozygous polymorphic genotype (CT) was higher in cases $65 \%(65 / 100)$ as compared to controls $32 \%(32 / 100)$. It was interesting to note that the frequency of the polymorphic homozygous genotype (CC) was higher in cases $15 \%(15 / 100)$ than controls $2 \%(2 / 100)$. While the frequency of the carrier genotype (CT + TT) was found to be more in cases $70 \%(70 / 100)$ than in controls $40 / 100$ $(40 \%)$. This study shows that $\mathrm{T}$ allele may be risk factor for reproductive tract infections as its percentage is higher in cases as compare to normal controls. Conclusion: TNF- $\alpha$ rs-281865419 locus may serve as an important biomarker for RTIs predisposition in Indian population though larger sample size is needed to validate the findings.

\section{Ovary: Poster Abstract}

\section{A rare case of ovarian and endometrial adenocarcinoma metastasized from carcinoma of jejunum \\ Sonam Agrawal, Pushpa Dahiya \\ PGIMS, Rohtak, Haryana, India}

Introduction: Krukenberg tumor of ovary is a rare clinical entity and accounts for $1-2 \%$ of all ovarian tumor. Stomach is most common primary site but other organs can also serve as a primary site. Accurate diagnosis of krukenberg tumor requires thorough endoscopic and histopathological examination to exclude primary tumor.

Case Report: 32 years old female presented with AUB for 2 months and history of jejunum carcinoma which was an incidental finding on biopsy after a surgery for intestinal obstruction. Endometrial biopsy showed endometrial carcinoma of mucin secreting signet ring type. CECT showed bilateral adnexal masses. Staging laparotomy was planned but due to dense adhesion and bladder and bowel infiltration optimal debulking could not be done and tumor was removed as much as possible. Patient was reffered for chemotherapy.

Conclusion: Krukenberg tumor is uncommon metastatic signet ring cell adenocarcinoma of ovary with transcoelomic spread. It is essential to rule out other ovarian malignancy to avoid the misdiagnosis and management of krukenberg tumor.

\section{Miscellaneous: Poster Abstract}

Prevalence of abnormal PAP smears in antenatal women in a tertiary hospital in India

Saritha Shamsunder ${ }^{1,2}$, Akriti Gautam ${ }^{1,2}$, Geetika Khanna ${ }^{1,2}$, Sunita Malik ${ }^{1,2}$

Departments of ${ }^{1}$ Gynecology and ${ }^{2}$ Pathology, Vardhman Mahavir Medical College and Safdarjung Hospital, New Delhi, India

Background: Pregnancy provides a good time for opportunistic screening of our women who rarely attend for cervical screening. The prevalence of abnormal PAP smear in pregnant women in developed countries was 5-6\%, however, no literature was available from India.

Aim: To determine the prevalence of abnormal PAP smears in antenatal women presenting to our antenatal clinic.

Methods: Women attending the antenatal clinic with gestation of $<28$ weeks were recruited after an informed consent and had a PAP smear by Ayre spatula and cytobrush or a broom type of cytobrush. The comfort level during smear taking $\&$ any problems noted were recorded using a pain score. The smears were stained using the PAP stain, were categorized as adequate or inadequate $\&$ classified as per Bethesda classification.

Results: We had 150 women participating, the mean age was $24.2 \mathrm{yrs}$, the mean period of gestation was 17 weeks; $43.9 \%$ were nulliparous. Smear adequacy rate was $71.5 \%$ overall. Pain during procedure was reported in $2.9 \%$ of women, $18.3 \%$ had minor discomfort; $78.6 \%$ were comfortable. Minor bleeding during smear taking was noted in 15\%; this was more with the cytobrush \& broom than the Ayre spatula alone. Abnormal smears were seen in three women; two had AGC \& one had LSIL.

Conclusions: Opportunistic cervical screening during pregnancy is safe and well tolerated. Abnormal Cervical smears were seen in $2 \%$ of our pregnant women.

\section{Miscellaneous: Poster Abstract}

\section{Inpatient pharmacologic management of malignant bowel} obstruction

\section{Rahul D. Arora}

Department of Palliative Medicine, Tata Memorial Centre, Tata Memorial Hospital, Mumbai, Maharashtra, India

Background: Management of life threatening complications encountered in Advanced Cancer is an important domain of Palliative Oncology. Malignant Bowel Obstruction is usually an indicator of poor prognosis in Advanced cancer. It is usually associated with malignancies in the gastrointestinal tract or those outside the gastrointestinal tract (gynaecological malignancies) $\mathrm{MBO}$ can also occur with primary peritoneal as well as secondary peritoneal malignancies. Diagnostic criteria for MBO include Clinical evidence of bowel obstruction, obstruction distal to the Ligament of Treitz, presence of primary intraabdominal or extra abdominal cancer with peritoneal involvement. Materials: Detailed below are two cases of Malignant Bowel obstruction managed with Conservative inpatient nonoperative management with discussion of the proposed pharmacological protocol for the same.

Case Details: A 45 year old Postmenopausal female diagnosed as carcinoma ovary stage iiic with left lower limb Deep Venous Thrombosis post multiple lines of chemotherapy including Paclitaxel plus Carboplatin, Etoposide, Tamoxifen and Liposomal Doxorubin, Malignant pleural effusion post thoracentesis was seen in the wards. A 31 year old Female a known case of moderately differentiated carcinoma colon with transmural infiltration and serosal seeding along with omental deposits with hepatic metastasis was seen in the casualty with signs of Multiple episodes of bilious vomiting with colicky abdominal pain and diagnosed to have malignant bowel obstruction on clinic radiological evaluation. Both these patients were provided non operative management of malignant bowel obstruction, were kept nil per oral, nasogastric decompression was performed with ryles tube insertion, antisecretory medication Inj Octreotide 100 ug three times daily, Ant Edema measures Inj Dexamethasone $8 \mathrm{mg}$ intravrenous three times daily, Anti spasmodic and anti secretory medication Inj Hyoscine Butyl bromide $10 \mathrm{mg}$ three times daily, inj Metronidazole $500 \mathrm{mg}$ intravenous three times daily and Pain medication Inj Tramadol hydrochloride $50 \mathrm{mg}$ intravenous 
in $100 \mathrm{ml}$ of normal saline three times daily. Both these patients developed hyperglycemia which was managed with human regular insulin prescribed as per the sliding scale.

Results: Ryles tube aspirate showed a decreasing trend and both the Patients achieved clinical resolution of symptoms underwent deintubation on Day 10 and Day 13 respectively and were taking oral feeds at the time of discharge. They were prescribed pharmacologic management of adhesive bowel obstruction consisting of Tab activated Dimethicone $40 \mathrm{mg}$ three times daily, Tab Lactobacillus one tablet three times daily and Polyethylene glycol one satchet upto three times daily for 15 days at the time of discharge. Results: Resolution of symptoms can be achieved by providing non operative pharmacological management outlined above which consists of adequate hydration, parenteral nutrition when indicated, antibiotics, decongestive anti edema measures, anti spasmodic and anti secretory medication.

Conclusion: Management of Hyperglycemia induced by Octreotide and Dexamethasone requires Insulin therapy. Optimum Duration, dosage and route of administration of Octreotide in management of Malignant Bowel Obstruction needs to be evaluated further.

Key words: Malignant bowel obstruction; octreotide; pharmacological management

\section{Miscellaneous: Poster Abstract Kanika Bajaj \\ MMIMSR, Mullana, Haryana, India}

Tuberous sclerosis (TS) is a genetic disorder that is inherited in an autosomal dominant fashion with variable clinical manifestations including seizures, mental retardation, renal failure and pneumothorax. The literature on TS in pregnancy is largely based upon case reports which have shown a 43\% complication rate including oligohydramnios, polyhydramnios, IUGR, hemorrhage from ruptured renal tumors, PPROM, renal failure, placental abruption and perinatal demise. We reporting a case of $33 \mathrm{yr}$ old female with gravida 3 para 2 and live 2 with period of gestation 9 months with tuberous sclerosis, with severe oligohydramnios with fetal cardiomegaly and mild pericardial effusion and pleural effusion. She had facial angiofibromas along with bilateral renal angiomyolipomas. The previous fetal outcomes were normal, with facial angiofibroma. We report such a unique case having all clinically diagnostic physical sings of tuberous sclerosis with good fetal outcomes.

\section{Miscellaneous: Poster Abstract}

Vaginal dilator therapy to prevent stenosis from radiotherapy: A systematic review

Nupur Bansal, Abhishek Soni, Anil Khurana, Yashpal Verma, Paramjeet Kaur, Ashok Kumar Chauhan

Department of Radiotherapy, Pt. B.D.S. PGIMS, Rohtak, Haryana, India

Background: Pelvic radiotherapy may damage the vagina and cause vaginal stenosis. Its incidence in the literature ranges from $1.2 \%$ to $88 \%$. To prevent vaginal stenosis, routine vaginal dilation is recommended during and after pelvic radiotherapy.

Materials and Methods: The objective was to examine critically the evidence behind this guideline. Searches included the Cochrane Central Register of Controlled Trials, MEDLINE, EMBASE and Google scholarly articles. All the relevant articles were included in the study.

Discussion: Various studies gave recommendations on dilation during or immediately after radiotherapy. Literature does not support routine vaginal dilatation during or immediately after pelvic radiotherapy. Occasional penetration might prevent the sides of the vagina adhering to each other, and dilation might be valuable once the inflammatory and psychological scarring has settled. Two trials demonstrated that encouraging vaginal dilation increased patient compliance, but no difference was found in sexual function scores in the first trial. One retrospective study reported that dilation lowered stenosis rates, but the control group is not comparable. One study involving 89 women revealed that the median vaginal length was $6 \mathrm{~cm}$, six to ten weeks after radiation therapy, but women tolerated a 9-cm dilator/measurer after 4 months of dilation experience. One trial showed no significant advantage by inserting mitomycin C. A study of five women reported that vaginal stenosis can be treated by dilation even many years after radiotherapy. Dilation during or immediately after radiotherapy can cause damage, and there is no evidence that it prevents stenosis. Dilation might stretch the vagina if commenced after the inflammatory phase. Dilation has been associated with traumatic rectovaginal fistulae and psychological consequences.

Conclusion: Vaginal dilation might help treat the late effects of radiotherapy, but it must not be assumed that this applies to the acute toxicity phase. Routine dilation during treatment is not supported by good evidence. Prophylactic and therapeutic dilation therapy needs to be considered separately and research is needed to determine when dilation therapy should start on a large population.

\section{Miscellaneous: Poster Abstract}

Collision tumor of endometrial stromal sarcoma and squamous cell cancer: A rare entity

Bindiya Gupta, Shalini Rajaram, Sandhya Jain, Neerja Goel, Naveen Tanwar

A collision tumor is defined by the presence of two separate tumors in one organ on gross, microscopic, and immunohistochemical studies and they should be distinguished from malignant mullerian mixed tumors. A 60 year old lady P8L8 presented with blood stained vaginal discharge and post menopausal bleeding. Examination revealed a $1 \times 2 \mathrm{~cm}$ cervical growth which was reported as squamous cell carcinoma cervix. Imaging revealed myohyperplasia with normal uterine cavity. The patient underwent Type III radical hysterectomy, bilateral salphingo-oophorectomy and bilateral pelvic lymphadenectomy. The uterine corpus revealed $5 \mathrm{~cm}$ growth in uterine cavity which was reported as high grade endometrial stromal sarcoma and the cervical growth was non keratinising squamous cell carcinoma infiltrating the former. The lymph nodes, parametria and vaginal cuff were free of tumor. The patient was referred for adjuvant chemotherapy and radiation therapy.

\section{Miscellaneous: Poster Abstract}

\section{Case series: Breast and ovarian cancer syndrome}

Aims and Objectives: To report a series of cases with breast and ovarian carcinomas either in same patient or in a family and identifying the importance of BRCA 1, 2 genetic testing in such individuals.

Materials and Methods: The medical records of breast and ovarian cancer patients operated over past 3 years at a single institute were reviewed retrospectively and their clinical profile, family history, final pathological reports and follow up data was collected.

Results: 8 patients were found to have breast and ovarian malignancies, out of which 3 had synchronous breast and ovarian cancers, 4 had metachronous and 1 patient with ovarian cancer had history of breast cancer in family. Median age of presentation to the hospital was 47 years and median time interval in metachronous disease patients was 5.5 years.

Conclusion: About $5 \%$ of people who have breast cancer and about $10 \%$ of women who have ovarian cancer have HBOC, caused by germline mutation in BRCA 1, 2 gene. These individuals have increased risk of developing breast cancer at younger age, TNBC, or developing a second primary in breast or ovary plus an overall risk of breast/ovarian/prostate/pancreatic malignancies in other family members due to inheritable mutation. Identification of BRCA mutation in such individuals can help family members to undergo genetic counseling and follow different screening and prevention guidelines from general population thus reducing the cancer risks.

\section{Miscellaneous: Poster Abstract}

\section{Female adnexal tumour of probable wolffian origin: A rare} case report

Nehal Dhaduk, Mamta Dagar, Mala Srivastava, Punita Bhardwaj, Tina Verma, Indrani Ganguli, Nayak Introduction: Female adnexal tumour of probable wolffian origin (FATWO), is a rare neoplasm arising within the leaves of a broad ligament or hanging from it or a fallopian tube. It is considered a tumour of low malignant 